\title{
Limbic encephalitis and antibodies to Ma2: a paraneoplastic presentation of breast cancer
}

\author{
Ian Sutton, John Winer, David Rowlands, Josep Dalmau
}

\begin{abstract}
A patient with atypical medullary breast cancer is described who presented with symptoms of limbic encephalitis. The patient's serum and CSF contained antibodies that reacted with the nervous system and the tumour. These antibodies recognised Ma2, a neuronal protein related to paraneoplastic limbic and brainstem encephalitis in men with testicular tumours. This report highlights the importance of testing for paraneoplastic antineuronal antibodies in cases of unexplained limbic encephalitis and suggests screening for breast cancer in women with antibodies predominantly directed to $\mathrm{Ma} 2$.

(F Neurol Neurosurg Psychiatry 2000;69:266-268)
\end{abstract}

Keywords: paraneoplastic; limbic encephalitis; anti$\mathrm{Ma} 2$ antibodies; breast cancer

Recent studies have shown that some patients with paraneoplastic neurological disorders develop antibodies against a novel family of brain-testis-cancer antigens, called $\mathrm{Ma}$ proteins. ${ }^{12}$ Detection of antibodies reacting only or almost exclusively with $\mathrm{Ma} 2$ (called anti-Ma2 or anti-Ta) usually associates with paraneoplastic limbic and brainstem encephalitis in men with germ cell tumours of the testis. We report on a woman with breast cancer, paraneoplastic limbic encephalitis, and serum and CSF antibodies almost exclusively directed to $\mathrm{Ma} 2$.

\section{Case history}

In February 1998 this 58 year old woman was referred to a general physician for assessment of cognitive dysfunction. The general examination showed a recurrent mass in the upper outer quadrant of the left breast. Seven years earlier the patient had undergone surgical removal of a mass from this site, which was reported as an incompletely excised complex sclerosing lesion showing epithelial hyperplasia without atypia. Fine needle aspiration of the recurrent lesion disclosed carcinomatous cells and histopathological examination of the subsequent wide local excision specimen and axillary lymph nodes confirmed complete resection of an atypical medullary tumour without nodal involvement. The patient was started on tamoxifen and received a course of adjuvant radiotherapy.

Postoperatively her cognitive dysfunction persisted and in May 1998 she was referred for neurological assessment. For 9 months the patient had been aware of an inability to remember recent events. Her family had noticed that the patient was no longer able to retain new information and colleagues at work had commented that she had become unable to perform her job as a wages clerk. There had been no deterioration in the patient's symptoms in the 3 months after surgery. There was no history of seizure activity, gait disturbance, personality change/psychiatric symptoms, hallucination, or sphincter dysfunction.

Cognitive assessment showed that the patient had a severe amnesic syndrome with markedly impaired anterograde verbal and visual memory. There was also retrograde amnesia for both semantic and episodic events extending back to about 1980. Implicit memory was unaffected and the neurological examination was otherwise unremarkable.

T1 weighted MRI studies showed bilateral atrophy of the mesial temporal lobes and limbic structures and on T2 weighted images (without gadolinium) there was signal change within both mesial temporal lobes (fig 1). The CSF

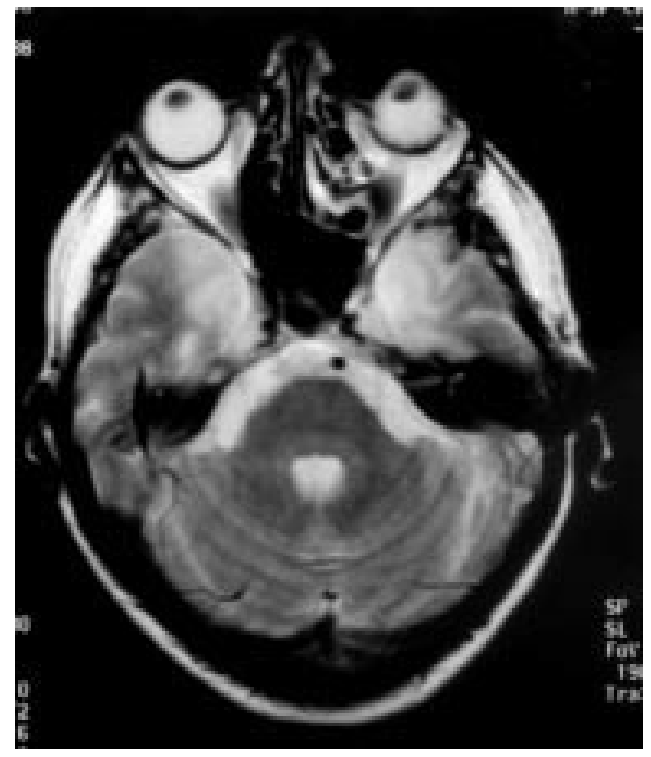

Figure $1 \quad T 2$ weighted MRI (without gadolinium) showing bilateral mesial temporal lobe signal change. 


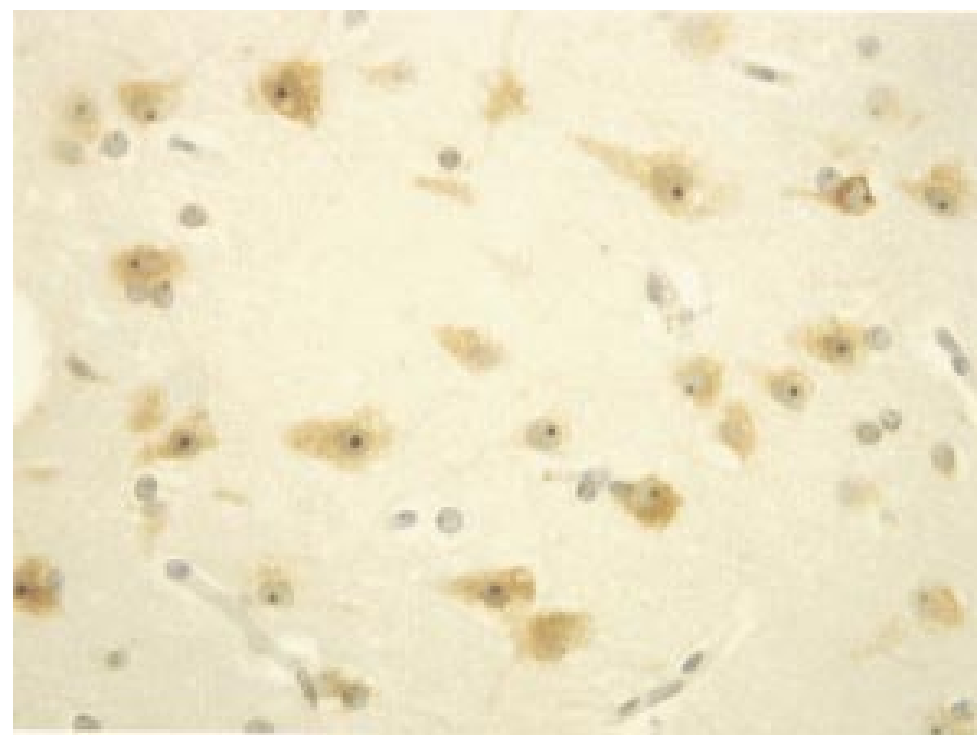

Figure 2 Photomicrograph (magnification $\times 400)$; indirect immunohistochemistry demonstrating that serum contains antineuronal antibodies. Reactivity is seen in the nucleoli, nuclei, and to a lesser extent the cytoplasm of neurons of the cerebral cortex (serum dilution 1:250).

contained $<1$ lymphocyte $/ \mathrm{mm}^{3}, 0.42 \mathrm{~g} / 1$ protein, and $3.9 \mathrm{mmol} / 1$ glucose. Cytology was negative. Oligoclonal bands were seen within the serum and CSF. Immunohistochemical and western blot analysis of the serum and CSF for the presence of antineuronal antibodies were done as previously reported. ${ }^{1}$ Immunohistochemistry demonstrated that the serum contained antineuronal antibodies at a titre of 1:500. Antibodies reacted predominantly with the nucleoli of neurons, with lesser reactivity within the nucleus and cytoplasm; there was no reactivity with glial cells (fig 2 ). Western blot of
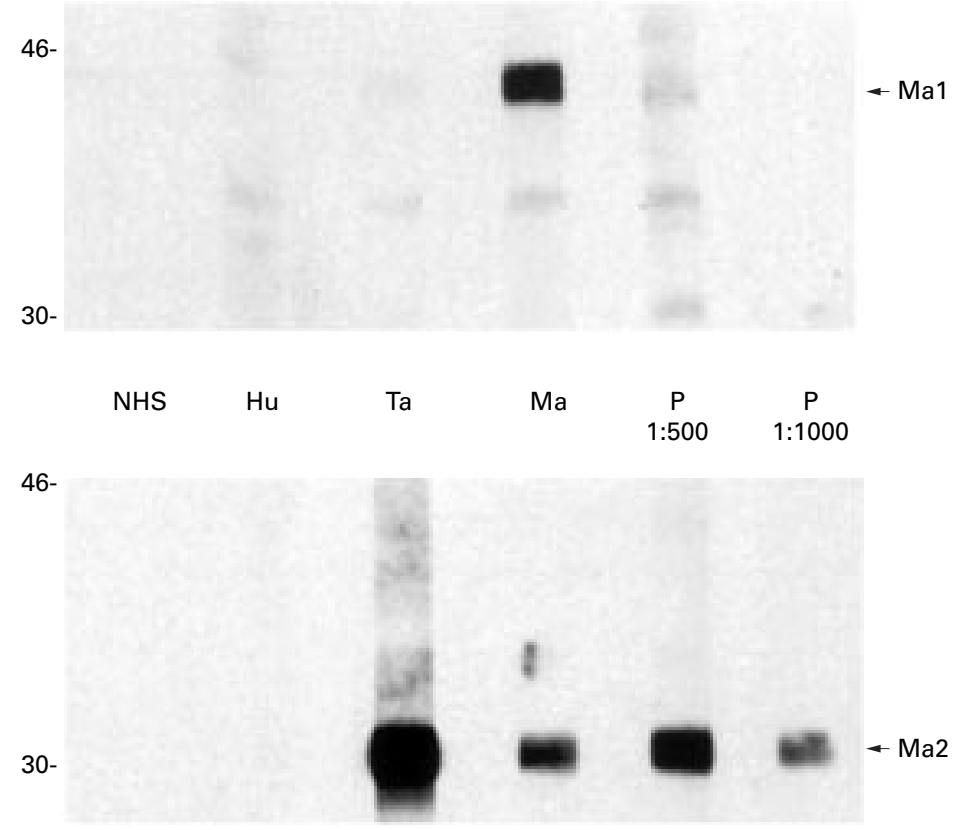

Figure 3 Western blot analysis of the patient's serum with Ma1 (top panel) and Ma2 (bottom panel) fusion proteins. The serum of the patient $(P)$ was examined at two dilutions (1:500) and (1:1000) and reacted with both Ma1 and Ma2, but with weaker reactivity with Ma1. Control sera: normal human serum (NHS) (1:500), anti-Hu (1:1000), anti-Ta (1:1000), anti-Ma (1:1000). Note that anti-Ma2 serum (also called anti-Ta) reacts with Ma2, and anti-Ma serum reacts equally with both Ma1 and Ma2. recombinant Ma proteins confirmed that both the serum and CSF contained antibodies that reacted with $\mathrm{Ma} 2$ and showed marginal reactivity with Ma1 (fig 3). In addition, the patient's biotinylated IgG reacted with the paraffin embedded tumour cells (fig 4); this reactivity was also detected using the biotinylated IgG from another patient with anti-Ma2 antibodies (data not shown), but not with biotinylated IgG from a normal person. Other investigations, including an EEG, were normal.

A clinical follow up in June 1999 confirmed that there had been no further deterioration in cognitive function. The patient had no evidence of tumour recurrence and anti-Ma antibodies were no longer detectable by immunohistochemistry.

\section{Discussion}

Limbic encephalitis can arise as a paraneoplastic complication of several different tumour types, but only five of the reported patients with paraneoplastic limbic encephalitis had an associated breast cancer. Most cases of paraneoplastic limbic encephalitis have occurred in association with small cell lung cancer and $50 \%$ are associated with anti-Hu antibodies. ${ }^{3}$

Recent studies showed that a novel family of brain-testis-cancer proteins, called $\mathrm{Ma}$ proteins, are target antigens of antibodies associated with paraneoplastic limbic and brainstem encephalitis, and cerebellar degeneration. ${ }^{12}$ Cumulative experience with 22 patients with immunity to Ma proteins (14 reported $^{12}$ and eight unpublished) suggests two patterns of serum reactivity. Firstly, a group of patients $(n=6)$ whose antibodies, called anti-Ma, react equally with both Ma1 and Ma2 proteins. The presence of these antibodies associates with paraneoplastic brainstem and cerebellar dysfunction and several types of cancer (lung, breast, colon, parotid). The second group of patients $(n=16)$ harbour antibodies, called anti-Ma2 or anti-Ta, that react only or almost exclusively with Ma2, sometimes showing marginal reactivity with Ma1. These antibodies are detected in patients with paraneoplastic limbic and brainstem encephalitis, and the associated tumour is usually a germ cell neoplasm located in the testis (15 germ cell tumours, one non-small cell lung cancer). In the current case, the pattern of antibody reactivity is similar to the latter group of patients, but the patient is a female with breast cancer. This finding suggests that Ma2 is a major autoantigen associated with limbic encephalitis irrespective of the tumour type.

It is not known whether antibodies against Ma proteins play a part in the pathogenesis of limbic encephalitis. However, other paraneoplastic antibodies reactive with nuclear antigens do not seem to be pathogenic. ${ }^{4}$ A CD8 positive lymphocytic infiltrate is seen in neuropathological studies of CNS paraneoplastic disorders ${ }^{5}$ and as under certain circumstances hippocampal cells upregulate $\mathrm{MHC}$ class I expression in vivo ${ }^{6}$ this raises the possibility that limbic encephalitis could be mediated by cytotoxic $\mathrm{T}$ lymphocytes. 


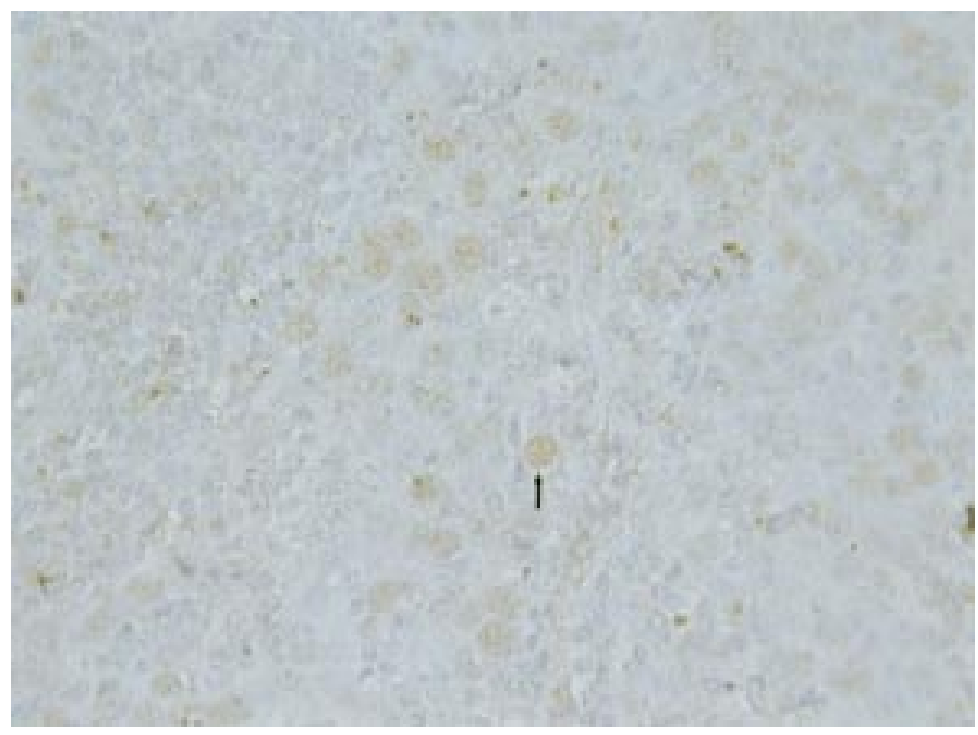

Figure 4 Photomicrograph (magnification $\times 400$ ); section of the patient's tumour incubated with biotinylated IgG isolated from the patient's serum. Direct immunohistochemistry demonstrates that the serum contains antibodies that react with tumour cells; reactivity involves predominantly the nucleus of the cells (arrow).

Ma protein expression is normally restricted to the brain and testis, but in this case the tumour of the patient expressed immunoreactive Ma proteins. Although complex sclerosing lesions are now recognised as a premalignant condition, ${ }^{7}$ the patient's initial complex sclerosing lesion was not available to examine for expression of Ma proteins. It is of note that breast cancer usually arises in the periphery of complex sclerosing lesions ${ }^{7}$ and in this case the surgical resection of the complex sclerosing lesion was incomplete. We postulate that in the course of transformation of the complex sclerosing lesion to carcinoma, expression of $\mathrm{Ma}$ antigens by the tumour led to breakdown in immune tolerance and subsequent immune mediated neuronal damage. This report stresses the importance of testing for antineuronal antibodies in cases of unexplained limbic encephalitis and that screening for breast cancer should be considered in patients seropositive for antibodies against the Ma proteins.

We thank Dr David Heath for referring the patient. IS is funded by a Sheldon Research Fellowship and The Midland Neurosciences Teaching Fund.

1 Dalmau J, Gultekin SH, Voltz R, et al. Ma1, a novel neuronand testis-specific protein, is recognised by the serum of patients with paraneoplastic neurological disorders. Brain 1999;122:27-39.

2 Voltz R, Gultekin SH, Rosenfeld MR, et al. A serologic marker of paraneoplastic limbic and brain stem encephalitis in patients with testicular cancer. N Engl F Med 1999;23: $1788-95$.

3 Alamowitch S, Graus F, Uchuya M, et al. Limbic encephalitis and small cell lung cancer: clinical and immunological is and small cell lung cancer:

4 Sillevis-Smitt PA, Manley GT, Posner JB. Immunization with the paraneoplastic antigen HuD does not cause with the paraneopiastic antigen HuD does not cause

5 Graus F, Ribalta T, Campo E, et al. Immunohistochemical Graus F, Ribalta T, Campo E, et al. Immunohistochemical
analysis of the immune reaction in the nervous system in paraneoplastic encephalomyelitis. Neurology 1990;40:21922

6 Corriveau RA, Huh GS, Shatz CJ. Regulation of class I MHC expression in the developing and mature CNS by neural activity. Neuron 1998;21:505-20.

7 Sloane JP, Mayers MM. Carcinoma and atypical hyperplasia in radial scars and complex sclerosing lesions: importance of lesion size and patient age. Histopathology 1993;23:22531 